\title{
A tiered approach to address regulatory drug metabolite-related issues in drug development
}

\author{
We believe, with the improvement in LC-MS technologies, the determination of circulating metabolite concentrations \\ in humans and animals will continue to evolve and there will be less dependency on the validated \\ bioanalytical methods to address regulatory issues.
}

Keywords: HRMS $₫$ metabolite identification $₫$ metabolite in safety testing $₫$ metabolite quantification $₫$ MIST $₫$ tiered approach

Identification and quantification of circulating metabolites in humans are required to address three important regulatory requirements: assessment of safety coverage of metabolites; understanding the drug-drug interaction (DDI) potential of metabolites; and determination of whether metabolites contribute significantly to the overall pharmacology of a drug. Recent regulatory guidance on metabolites in safety testing (MIST) [1,2] requires the identification of human metabolites at steady state and determination of whether major circulating metabolites ( $>10 \%$ of total drug-derived material) are present at equal or greater exposure in at least one of the toxicology species used in safety testing. The DDI guidance also proposes that human metabolites present at $\geq 25 \%$ of the parent AUC and/or $>10 \%$ of total drug-derived material, should be investigated in vitro for their DDI potential [3,4]. The European Medicines Agency DDI guidance recommends that the enzymes involved in the formation and elimination pathway of those metabolites that contribute to $\geq 50 \%$ of the in vivo target pharmacological effect, should be identified [3]. These regulatory guidance documents have highlighted not only the importance of the identification of drug metabolites, but also the determination of their quantity early in clinical development. The timing and the rigor with which the determinations are to be made (e.g., semi-quantitative method vs validated method) for metabolite quantification are based on the stage of development and issues at hand for a given development program. Therefore, a tiered approach is appropriate from the business points of view, as well as to address scientific questions at specific stages of drug development.

To determine whether any metabolite falls into MIST or DDI considerations, human circulating metabolites must first be characterized.
In recent years, the pharmaceutical industry has assessed human metabolism at a steady state by profiling plasma samples typically from Phase I multiple ascending dose (MAD) studies. The advances in HRMS technologies and the development of 'intelligent' data processing tools in the last decade have greatly improved the analytical capabilities to detect and identify metabolites with ease from nonradiolabeled first-in-human studies $[5,6]$.

The decision on when and how to measure drug metabolites during clinical development may be dependent on the amount (triggering safety coverage requirement) and the nature of the metabolite, for example, stable metabolites with no pharmacological activity, metabolites that possess significant pharmacological activity or reactive metabolites that may contribute to off-target toxicity. Validated analytical methods need authentic standards and take time to develop and validate. There is a considerable anxiety over when these methodologies are needed. Recently, the European Bioanalysis Forum provided a recommendation on a tiered approach involving three levels of method validation and data quality, defined as screening methods, qualified methods and validated methods, to be used for metabolite quantification when assessing the relative abundance or absolute concentrations of metabolites during drug development [7]. The 'screening method', which is often referred as 'semi-quantitation method', can provide reliable quantitation of human metabolites using UV or radioactivity correction for mass spectrometric response [8,9] or NMR [10] and can serve the purpose for early clinical metabolite investigation. The circulating metabolite levels will be confirmed from a ${ }^{14} \mathrm{C}$-ADME study later in the development program if the program progresses deep into

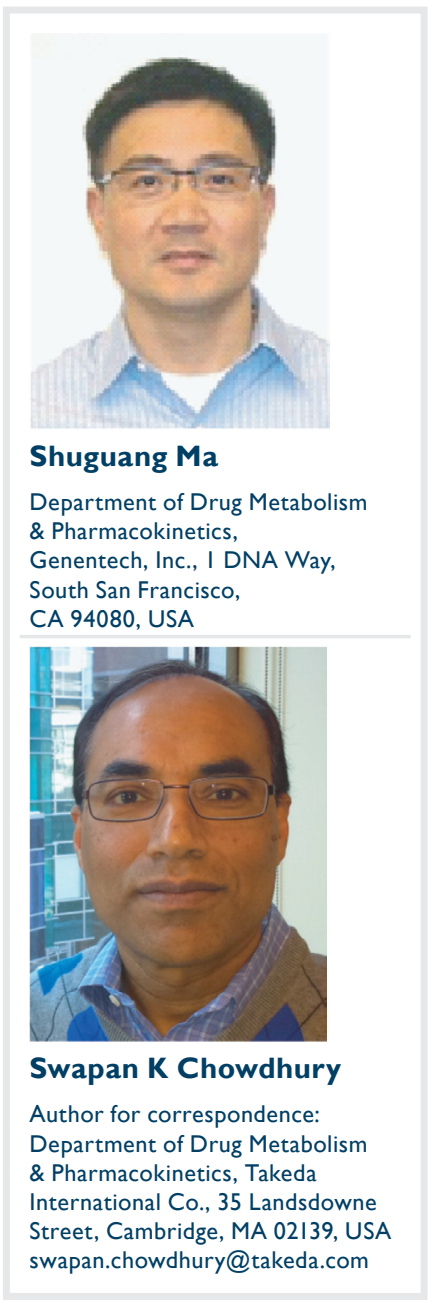

FUTURE
SCIENCE FSS 
clinical development. The utility of human ${ }^{14} \mathrm{C}$-ADME study $[11,12]$ in generating metabolite concentration data has not been emphasized in the European Bioanalysis Forum's White Paper and should be considered as an important quantitation step in the overall approach to address regulatory issues. Human ${ }^{14} \mathrm{C}$-ADME study is the most reliable and perhaps the only source for information on metabolite level as percentage of total drug-related material in circulation and, in some cases, could be a sufficient confirmation of human metabolite levels to obviate the need for qualified or validated analytical methods. When the synthesis of reference standards is difficult or not possible, ADME studies could provide an avenue to assess human metabolite coverage. Although the human ADME study is most often a single dose study, from PK considerations, steady state AUC for a given dose interval $(\tau)$ is equal to the AUC infinity for single dose.

$$
\mathrm{AUC}_{0-\tau}^{\mathrm{SS}}=\mathrm{AUC}_{0-\infty}^{\mathrm{SD}}
$$

Equation I

Therefore, with appropriate time-averagedAUC pooling of human samples, metabolite profiling data can provide quantitative information on the metabolite levels expected at steady state, which was demonstrated by Prakash et al. [12].

\section{Proposed tiered approach}

- Stable metabolites of MIST concern

The MIST guidance does not require the knowledge of the actual exposures of circulating metabolites, as long as these metabolites are adequately exposed in any one of the preclinical safety species. Based on this concept, simple and reliable LC-MS methods were developed for semi-quantitative assessment of relative exposures to metabolites by comparing the peak area ratios of the metabolites in animals to humans from MRM experiments [13] or high-resolution full scan accurate MS [14]. These quantitation methods have adequate accuracy and precision and should be sufficient to demonstrate metabolite's exposure coverage in complying with MIST guidance. Characterization of a metabolite's toxicity risk would generally be considered adequate when animal exposure is at least $50 \%$ of the exposure seen in humans at intended clinical dose [15]. Only in rare cases where animals have lower exposure to the metabolite and if toxicology studies are needed by direct administration of the metabolite, will an actual exposure measurement of a metabolite with a validated bioanalytical method be necessary [16].
Stable metabolites of DDI concern Both the US FDA and European Medicines Agency require in vitro assessment for DDI potential of circulating metabolites at $\geq 25 \%$ of the parent compounds' exposures [17]. The relative exposure of metabolite can be obtained from the metabolite profiling of time-averaged AUC-pooled human plasma sample from a MAD study, after applying a MS response factor of the metabolite relative to the parent $[6,16]$. These data should provide sufficient information before definitive data obtained from the human ${ }^{14} \mathrm{C}-\mathrm{ADME}$ study.

\section{- Metabolites with significant pharmacological activity}

In general, if a metabolite is expected to contribute $\geq 50 \%$ of the overall pharmacological effect of the drug, routine quantitation will be needed to ascertain its impact on the PD response. Whether a qualified method or a validated method should be employed during toxicology and clinical studies depends on the specific program needs and should be treated on a case-by-case basis. If the regulatory authorities are expected to use the information to determine the drug's safety and efficacy, make dosing instructions, or indicate patient treatment, then the methods for generating these data may be required to be fully validated $[15,18]$.

\section{Reactive metabolites of safety concern}

Highly chemically reactive metabolites, while of toxicological concern, do not generally circulate in plasma due to their short half-life and, most often, the byproducts are the only species that can be detected. Since it is not feasible to test highly reactive metabolites independently, the formation of downstream products, such as mercapturic acids, dihydrodiols, methyl catechols and conjugates of dihydroquinones, are indicative of the presence of a reactive intermediate, but sometimes may give misleading information regarding the actual amount of reactive metabolite formation. Their evaluations should have the same general considerations as any stable metabolite.

In summary, we recommend the following tiered approaches in different scenarios to address drug metabolite-related regulatory issues in drug development:

- Scenario 1: No metabolite is greater than $>10 \%$ of total or $\geq 25 \%$ of parent estimated from MAD or human ${ }^{14} \mathrm{C}$-ADME study. No metabolite's activity is expected to be $\geq 50 \%$ of that of the parent. There are no structural 
alerts. Recommended actions - no further work is needed;

- Scenario 2: One or multiple metabolites are potentially $>10 \%$ of total or $\geq 25 \%$ of the parent drug. Recommended actions - perform safety coverage assessment using semi-quantitative methods [13,14]. Confirm metabolite levels from human ${ }^{14} \mathrm{C}$-ADME study. Explore metabolite synthesis and scale-up. Assess in vitro DDI liability of major metabolites. Determine pharmacological activity of major metabolites, if not known;

- Scenario 3: Human metabolites that are $>10 \%$ of total have safety coverage. There is no DDI risk for metabolites $\geq 25 \%$ of the parent drug. No metabolite contributes $\geq 50 \%$ of target pharmacological activity. Recommended actions - no further work is necessary. Discuss with regulators at appropriate time if metaboliterelated risks have been adequately addressed;

- Scenario 4: One or multiple metabolites contribute $\geq 50 \%$ of pharmacological activity. Disproportionate human metabolites are identified. Recommended actions - begin conversation with regulators on the path forward with safety assessment of metabolites. Develop and validate bioanalytical method(s) for metabolite quantitation. Obtain definitive metabolite concentration in safety studies or surrogate studies with appropriate dose and in clinical samples.

\section{Future perspective}

We believe, with the improvement in LC-MS technologies, the determination of circulating metabolite concentrations in humans and animals will continue to evolve and there will be less dependency on the validated bioanalytical methods to address regulatory issues. As more data becomes available on DDI caused by major metabolites through retrospective analysis, there will be a better understanding on the relevant amount to trigger the evaluation of metabolites for DDI [19]. With the increase of familiarity, the use of accelerator MS technologies will continue to increase in microtracer-dosed clinical studies as an alternative approach to detect and quantify human metabolites early in clinical-development programs [20]. Inclusion of a low radioactive ${ }^{14} \mathrm{C}$-dose $(-100 \mathrm{nCi})$ on top of the therapeutic nonradioactive dose can be administrated to healthy volunteers during conventional first-inhuman studies. All these approaches could ease the burden on the need for validated analytical methods for metabolite quantitation, inorder to address regulatory issues in drug development.

Financial \& competing interests disclosure

The authors have no relevant affiliations or financial involvement with any organization or entity with a financial interest in or financial conflict with the subject matter or materials discussed in the manuscript. This includes employment, consultancies, honoraria, stock ownership or options, expert testimony, grants or patents received or pending, or royalties. No writing assistance was utilized in the production of this manuscript.

\section{References}

1 US FDA. Guidance for Industry: Safety Testing of Drug Metabolites (2008). www.fda.gov/downloads/Drugs/ GuidanceComplianceRegulatoryInformation/ Guidances/ucm079266.pdf

2 International Conference on Harmonisation. Guidance on nonclinical safety studies for the conduct of human clinical trials and marketing authorization for pharmaceuticals M3(R2) (2009).

http://private.ich.org/LOB/media/ MEDIA5544.pdf

3 European Medicines Agency. Guideline on Investigation of Drug Interactions (2012). www.ema.europa.eu/docs/en_GB/ document_library/Scientific_ guideline/2012/07/WC500129606.pdf

4 US FDA. Guidance for Industry: Drug Interaction Studies - Study Design, Data
Analysis, Implications for Dosing, and Labeling Recommendations (2012). www.fda.gov/downloads/Drugs/ GuidanceComplianceRegulatoryInformation/ Guidances/ucm292362.pdf

5 Zhu M, Zhang H, Humphreys WG. Drug metabolite profiling and identification by high-resolution mass spectrometry. J. Biol. Chem. 286(29), 25419-25425 (2011).

6 Ma S, Chowdhury SK. Analytical strategies for assessment of human metabolites in preclinical safety testing. Anal. Chem. 83(13), 5028-5036 (2011).

7 Timmerman P, Anders Kall M, Gordon B et al. Best practices in a tiered approach to metabolite quantification: views and recommendations of the European Bioanalysis Forum. Bioanalysis 2(7), 1185-1194 (2010).
8 Yang Y, Grubb MF, Luk CE et al. Quantitative estimation of circulating metabolites without synthetic standards by ultra-high-performance liquid chromatography/high resolution accurate mass spectrometry in combination with UV correction. Rapid Commun. Mass Spectrom. 25(21), 3245-3251 (2011).

9 Yi P, Luffer-Atlas D. A radiocalibration method with pseudo internal standard to estimate circulating metabolite concentrations. Bioanalysis 2(7), 1195-1210 (2010).

10 Vishwanathan K, Babalola K, Wang J et al. Obtaining exposures of metabolites in preclinical species through plasma pooling and quantitative NMR: addressing metabolites in safety testing (MIST) guidance without using radiolabeled compounds and 


\section{EDITORIAL | Ma \& Chowdhury}

chemically synthesized metabolite standards. Chem. Res. Toxicol. 22(2), 311-322 (2009).

11 Penner N, Klunk LJ, Prakash C. Human radiolabeled mass balance studies: objectives, utilities and limitations. Biopharm. Drug Dispos. 30(4), 185-203 (2009).

12 Prakash C, Li Z, Orlandi C et al. Assessment of exposure of metabolites in preclinical species and humans at steady state from the single-dose radiolabeled absorption, distribution, metabolism, and excretion studies: a case study. Drug Metab. Dispos. 40(7), 1308-1320 (2012).

13 Gao H, Deng S, Obach RS. A simple liquid chromatography-tandem mass spectrometry method to determine relative plasma exposures of drug metabolites across species for metabolite safety assessments. Drug Metab. Dispos. 38(12), 2147-2156 (2010).
14 Ma S, Li Z, Lee KJ et al. Determination of exposure multiples of human metabolites for MIST assessment in preclinical safety species without using reference standards or radiolabeled compounds. Chem. Res. Toxicol. 23(12), 1871-1873 (2010).

15 Gao H, Jacobs A, White RE et al. Meeting report: metabolites in safety testing (MIST) symposium - safety assessment of human metabolites: what's really necessary to ascertain exposure coverage in safety tests? AAPS J. 15(4), 970-973 (2013).

16 Gao H, Obach RS. Addressing MIST (Metabolites in Safety Testing): bioanalytical approaches to address metabolite exposures in humans and animals. Curr. Drug Metab. 12(6), 578-586 (2011).

17 Yu H, Tweedie D. A perspective on the contribution of metabolites to drug-drug interaction potential: the need to consider both circulating levels and inhibition potency. Drug Metab. Dispos. 41(3), 536-540 (2013).

18 Smith G. Are multimetabolite methods fitfor-purpose for supporting toxicology studies? Bioanalysis 3(24), 2701-2704 (2011).

19 Callegari E, Kalgutkar AS, Leung L et al. Drug metabolites as cytochrome $\mathrm{p} 450$ inhibitors: a retrospective analysis and proposed algorithm for evaluation of the pharmacokinetic interaction potential of metabolites in drug discovery and development. Drug Metab. Dispos. 41(12), 2047-2055 (2013).

20 Lappin G, Seymour M. Addressing metabolite safety during first-in-man studies using ${ }^{14} \mathrm{C}$-labeled drug and accelerator mass spectrometry. Bioanalysis 2(7), 1315-1324 (2010). 